\title{
BIOLOGIA DA POLINIZAÇÃO E SISTEMA REPRODUTIVO DE Passiflora coccinea AUBL. EM MANAUS, AMAZONAS, BRASIL'.
}

\author{
Eliana Fernandez STORTI ${ }^{2}$
}

\begin{abstract}
RESUMO - Foi estudada a biologia da polinização e o sistema reprodutivo de Passiflora coccinea uma Passifloraceae comum na região Amazônica, conhecida popularmente por maracujá-poranga ou tomé-assu. Este estudo foi realizado em uma área perturbada, na Estação Experimental de

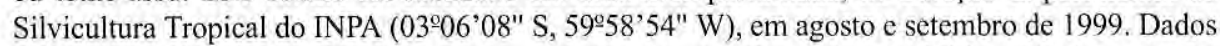
de morfometria, horário de abertura e tempo de vida das flores foram obtidos. Diariamente foram observados os animais visitantes, o tipo de alimento procurado, horário e freqüência das visitas às flores. Foram realizadas experiências sobre o sistema de reprodução, como também, observada a produção natural de frutos na área. A flor de $P$. coccinea apresenta a síndrome da ornitofilia pois tem antese diurna, é vermelha, não possui odor e apresenta néctar em abundância. Os beija-flores Amazilia sp. e Phaethornis superciliosus foram considerados seus polinizadores. Duas espécies de Hymenoptera e duas de Lepidoptera foram consideradas "ladras de pólen" e "ladras de néctar", respectivamente, pois não contactam a superfície estigmatífera. O sistema de reprodução desta espécie de maracujá é a xenogamia visto que não foram produzidos frutos por apomixia e por autogámia. A taxa natural de produção de frutos aumentou de $15 \%$ para aproximadamente $54 \%$ com a realização de polinizações manuais.
\end{abstract}

Palavras-chave: beija-flores, biologia floral, Passifloraceae.

Pollination Biology and Breeding System of Passiflora coccinea Aubl. in Manaus, Amazonas, Brazil.

ABSTRACT - Pollination biology and breeding system of Passiflora coccinea, a Passifloraceae common in the Amazonian region, popularly known as maracujà-poranga or tomé-assu, were studied in a clearing at the INPA's forestry experimental station $\left(03^{\circ} 06^{\prime} 08^{\prime \prime} \mathrm{S}, 59^{\circ} 58^{\prime} 54^{\prime \prime} \mathrm{W}\right)$ in August and September 1999. Data were obtained on floral morphology, blooming time and life span of the flower. Visiting animals, kind of foods taken, visiting time and frequency were observed daily. Experiments were carried out on the breeding system and the natural production of the fruits in the area was noted. The flower of $P$. coccinea presents a syndrome of the ornithophily since it has diurnal anthesis, is red, does not possess any odour, and presents large amounts of nectar. The hummingbirds Amazilia sp, and Phaethornis superciliosus were considered to be its pollinators. Two species of Hymenoptera and Lepidoptera were considered to be pollen and nectar "robbers", respectively, since they did not touch the stigma surface. Xenogamy is this maracuja species' breeding system, since fruits were not produced through autogamy and apomixis. Natural fruit production increased from 15 to nearly $54 \%$ when hand pollination was performed.

Key-words: flower biology, hummingbirds, Passifloraceae.

\section{Introdução}

\section{A familia Passifloraceae} apresenta aproximadamente 600 espécies e 20 gêneros de distribuição tropical e subtropical, principalmente nas Américas e África. O único gênero comercialmente importante da família é Passiflora, o qual apresenta algumas espécies que são cultivadas pelos seus frutos comestíveis ou como plantas ornamentais (Heywood, 1985).

\footnotetext{
'Trabalho desenvolvido durante o curso BOT 60 - Biologia Reprodutiva do INPA, de 16 a 30/08/99.

${ }^{2}$ Coordenação de Pesquisas em Ecologia - INPA. storti@inpa.gov.br
} 
Passiflora coccinea Aubl. é uma espécie conhecida popularmente por maracujá-poranga (Pio Corrêa, 1978) ou tomé-assu. Ela ocorre nas Guianas, Venezuela, Peru, Bolívia e Brasil (Amazônia). Produz frutos prontamente quando polinizada manualmente e estes frutos se tornam mais atrativos sendo apreciados, principalmente, na Guiana e em Guadalupe (Vanderplank, 1996).

A polinização de espécies de Passiflora foi estudada por Sazima \& Sazima $(1978 ; 1987)$ com $P$. mucronata (quiropterófila), Amela Garcia \& Hoc (1998) com P. foetida (melitófila) e, Sazima \& Sazima (1989) com P. edulis (melitófila), cuja presença de Trigona spinipes, abelha considerada pilhadora para esta espécie de maracujá, causa uma redução nas visitas das abelhas polinizadoras Xylocopa suspecta e X. frontalis, diminuindo assim, a produção de seus frutos. Da Silva et al. (1999) estudando P. edulis verificaram que o número de flores em antese e os fatores climáticos infuenciam a atividade polinizadora e a densidade populacional das abelhas Xylocopa.

O objetivo deste trabalho é conhecer a biologia floral e o sistema reprodutivo de Passiflora coccinea, por ser uma espécie de ampla distribuição na Amazônia brasileira e por seu potencial como planta comestivel.

\section{Material e Métodos}

Este estudo foi realizado em uma área perturbada na Estação Experi- mental de Silvicultura Tropical do INPA, no $\mathrm{km} 44$ da rodovia BR174 $\left(03^{\circ} 06^{\prime} 08^{\prime \prime} \mathrm{S}, 59^{\circ} 58^{\prime} 54^{\prime \prime} \mathrm{W}\right.$; a .37 metros do nível do mar). O período de estudo foi de agosto a setembro de 1999 , sendo que a temperatura variou de 20,5 a $33{ }^{\circ} \mathrm{C}$, com média de $26{ }^{\circ} \mathrm{C}$ e umidade entre 52 a $95 \%$, com média de $78 \%$.

Dez flores de Passiflora coccinea foram utilizadas para obtenção dos dados de morfometria e seus ovários examinados para contagem do número de óvulos. Amostras da planta foram coletadas para herborização e identificação. $\mathrm{O}$ horário de antese das flores e o seu tempo de vida foram observados. A receptividade do estigma foi observada com lupa manual, de $10 \mathrm{x}$ de aumento usando-se água oxigenada 3 $\%$, segundo a técnica de Arnold (1982).

A concentração de açúcares no néctar das flores de Passiflora coccinea foi obtida usando-se um refratômetro de bolso (Dafni, 1992) e a concentração de glicose no néctar foi verificada utilizando-se tiras reagentes Diastix, cf. Dra. Marlies Sazima com. pessoal.

$\mathrm{O}$ teor de produção de néctar foi verificado utilizando-se microcapilares de $20 \mathrm{ml} \mathrm{em} 20$ flores, das quais mediuse o diâmetro da corona. Para a conversão de milímetros $(\mathrm{mm})$ de néctar nos microcapilares em volume de néctar utilizou-se a seguinte fórmula de Cruden \& Hermann (1983):

$(\mathrm{a} / \mathrm{b}) \times \mathrm{c}=\mathrm{d}$, onde,

$\mathrm{a}=\mathrm{mm}$ de néctar na pipeta;

$\mathrm{b}=\mathrm{mm}$ equivalente do volume;

$\mathrm{c}=$ volume calibrado da pipeta $; \mathrm{e}$,

$\mathrm{d}=$ volume de néctar.

Foram feitas lâminas de pólen de 
algumas flores, para verificar a sua morfologia. Também se observou a porcentagem de grãos de pólen viáveis utilizando-se o azul de algodão em lactofenol (Radford et al., 1974) a cada hora, desde o momento da deiscência das anteras até o fechamento das flores.

A reflexão de raios ultra-violeta foi observada através do teste com hidróxido de amônia (Gertz, 1938) e com o uso de um aparelho com lâmpada ultra-violeta.

Diariamente eram observados os animais visitantes, o tipo de alimento procurado, horário e freqüência das visitas às flores. Alguns visitantes foram capturados com auxílio de um frasco matador contendo éter ou um puçá e fixados à seco para posterior identificação. Duas redes de captura de aves foram colocadas em locais próximos às flores de Passiflora coccinea com a finalidade de coletar os beija-flores visitantes, para melhor observação.

Para as experiências sobre o sistema de reprodução de Passiflora coccinea utilizou-se sacos de filó de malha fina $(<1 \mathrm{~mm})$. Para as experiências de autogamia espontânea, autogamia induzida, xenogamia interpopulacional, xenogamia intrapopulacional e apomixia foram utilizadas 37 flores recém abertas, nas quais o estigma estava receptivo; outras 37 flores foram apenas marcadas e utilizadas como controle. As flores utilizadas para a apomixia foram emasculadas. Todas as flores testadas receberam uma etiqueta numerada em ordem seqüencial com a data e a experiência realizada. Para o teste de xenogamia interpopulacional foi usado pólen de flores coletadas na margem da estrada, a aproximadamente $5 \mathrm{~km}$ de distância da área de estudo.

Os frutos resultantes das experiências sobre o sistema de reprodução de Passiflora coccinea foram pesados, medidos e anotado o número de sementes formadas em cada um deles. Em outros quatro frutos coletados na área de estudo realizou-se o mesmo procedimento.

Foram contados, ao acaso, 50 ramos para se verificar a produção natural de frutos.

Fotografias do local de estudo, da planta, flores, animais visitantes e de seus comportamentos durante as visitas às flores de Passiflora coccinea foram obtidas com o auxílio de uma máquina fotográfica Asahi Pentax, com objetiva macro de $50 \mathrm{~mm}$ e com câmera digital Canon PowerShot S100.

Amostras da planta encontramse depositadas no Herbário do Instituto Nacional de Pesquisas da Amazônia e registradas com o número 199.796.

Um exemplar de Heliconius burneyi encontra-se depositado na Coleção Entomológica do INPA.

\section{Resultados e Discussão}

Passiflora coccinea possui brácteas ovais de $5,16 \mathrm{~cm} \pm 0,54$ de comprimento, côncavas, glandulosas na margem, vermelhas ou alaranjadas. Suas sépalas (5) são linear-lanceoladas de $4,82 \mathrm{~cm} \pm 0,24$ de comprimento, vermelhas, cuculadas, carenadas no 
dorso, carena terminada por uma arista. As pétalas (5) são lineares de $5,24 \mathrm{~cm} \pm 0,19$ e vermelhas. Anteras (5) de deiscência, longitudinal, dorsifixas e versáteis com filetes de $1,53 \mathrm{~cm} \pm 0,16$ de comprimento. $O$ ovário é súpero, ovóide tomentoso, possui 3 estigmas com estiletes de $1,67 \mathrm{~cm} \pm 0,16$ de comprimento. Os ovários das dez flores examinadas apresentaram uma média de $437 \pm$ 87,7 óvulos. O androginóforo tem 5,1 $\mathrm{cm} \pm 0,17$ de comprimento. A corona de estaminódios é formada por filamentos que variaram da cor branca a vermelha com diâmetro de $1,44 \mathrm{~cm}$ $\pm 0,10$ (Fig. 1).

A antese das flores se dá por volta das 05:00 h, começando a se fechar às 09:00 h. No momento da abertura as anteras já se encontram deiscentes. Os movimentos florais ocorrem de modo semelhante aos de P. mucronata, descritos por Sazima e Sazima (1978).

A receptividade dos estigmas não é facilmente percebida e com a utilização da água oxigenada constatou-se que às $07: 00 \mathrm{~h}$ estes encontravam-se receptivos e após às 09:00 h não mais havia receptividade.

A média da concentração de açúcares no néctar das flores de $P$. coccinea foi de $35,1 \% \pm 1,9$. Esta média está próxima à encontrada por Amela Garcia \& Hoc (1998) que verificaram uma concentração de $34 \%$ em $P$. foetida. Segundo Percival (1974) os néctares mais concentrados atraem polinizadores mais especializados como os beija-flores e as borboletas. Com a utilização das tiras de Diastix verificou-se que o néctar destas flores apresentou uma

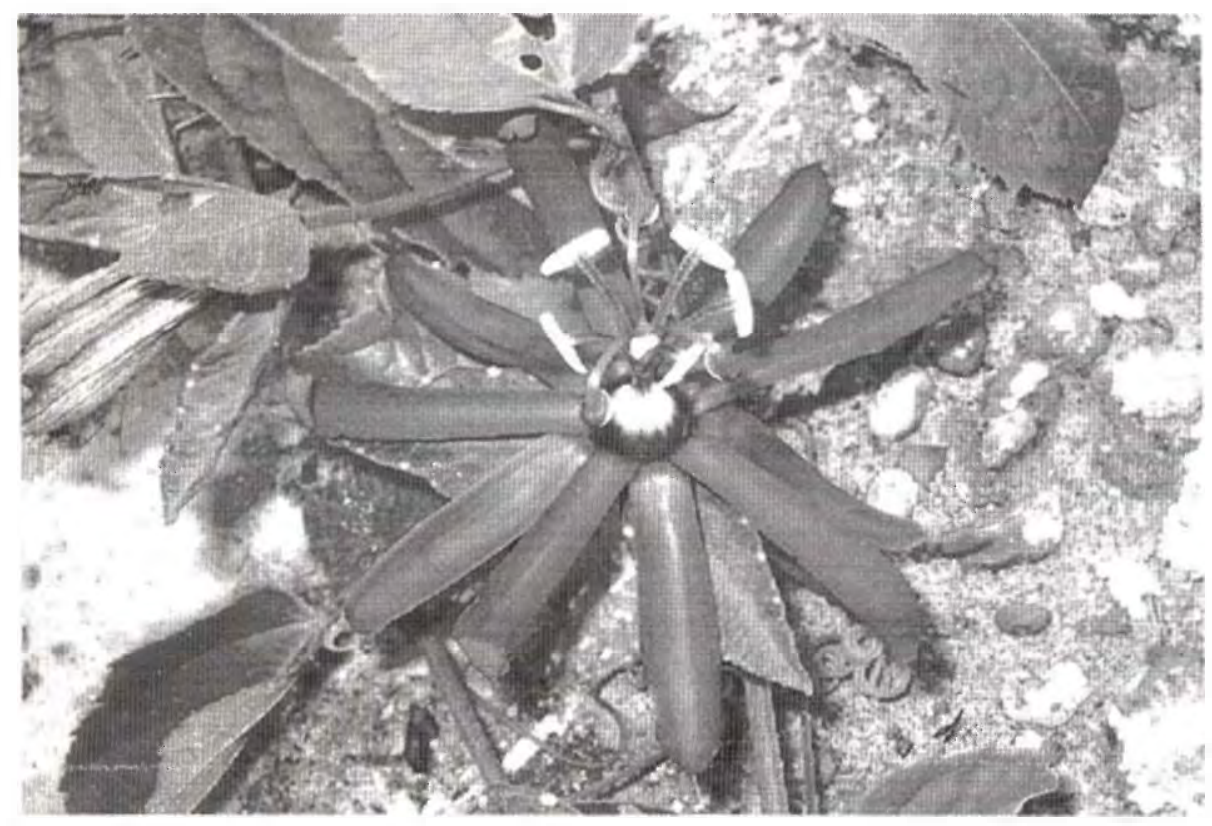

Figura 1. Flor de Passiflora coccinea Aubl. 
concentração de glicose de aproximadamente $500 \mathrm{mg} / 100 \mathrm{ml}$.

Das 20 flores observadas para relacionar a taxa de produção de néctar com o diâmetro da corona verificou-se que oito não continham néctar e, nas demais, o volume variou de 7,6 - 216,6 $\mu$ l, com uma média de $80,9 \pm 76,3 \mu$ l. Fischer \& Leal (1996) trabalhando com esta espécie de Passiflora em uma região próxima ao local deste estudo verificaram que a taxa de produção do néctar de flores individuais varia em função do diâmetro do cálice. Outras medidas devem ser obtidas para um melhor entendimento dos resultados e, também, verificarmos se existe alguma relação entre as cores da corona e a taxa de produção de néctar.

Os grãos de pólen desta espécie de maracujá apresentam superfície reticulada. Segundo Carreira (1978) eles são isopolares, de simetria radial, 6-colporados, de superficie reticulada com lúmens livres. Suas dimensões são: eixo polar $P=50-60 \mathrm{~mm}$ e eixo equatorial $E=65 \mathrm{~mm}$. Com a utilização do azul de algodão $\mathrm{em}$ lactofenol verificou-se que às $06: 00 \mathrm{~h}$ $100 \%$ dos grãos encontravam-se

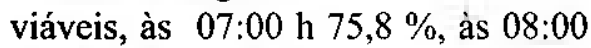
h 76,5\%, às 09:00 h 68,2 \%, sendo que a quantidade de pólen das anteras diminuia a cada uma destas amostras e, às 10:00 h elas já não apresentavam mais grãos de pólen.

Com a utilização do hidróxido de amônia verificou-se que as pétalas, sépalas e ápice dos estaminódios da corona coraram-se intensamente e, com a utilização da lâmpada ultra- violeta a base das pétalas e sépalas, corona, filetes, estiletes e nectários extraflorais apresentaram coloração branca indicando que estas áreas estào refletindo raios ultra-violeta.

Individualmente as plantas de Passiflora coccinea não apresentam grande quantidade de flores por dia. Tais flores nào exalam odor. Foi estimado que aproximadamente 15 flores ficavam bem expostas em toda a área de estudo e é a visão à curta distância que atrai os animais visitantes.

As flores desta planta foram visitadas por duas espécies de aves da ordem Apodiformes, familia Trochilidae: Amazilia sp. e Phaethornis superciliosus (Dr. Renato Cintra, com. pessoal), duas espécies de Hymenoptera: Melipona rufiventris e Trigona cf. spinipes (Plácido Oliveira, com. pessoal) e duas espécies de Lepidoptera: Heliconius burneyi Hübner, 1816 e uma espécie de Hesperiidae, não coletada (Tab. 1). Houve diminuição na freqüência das visitas no segundo dia de observação, provavelmente, pela diminuição de temperatura e aumento da umidade relativa do ar, devido a chuva durante a abertura das flores.

Ao visitarem as flores, no periodo entre 05:45 c 08:20 h, as duas espécies de beija-flor coletam o néctar, explorando todo nectário, que encontra-se protegido na corona e contactando os estigmas e anteras com a parte dorsal da cabeça. Estes beijaflores foram considerados polinizadores de Passiflora coccinea pelo seu comportamento ao visitarem 
Tabela 1. Visitantes florais de Passiflora coccinea, alimento procurado e freqüência de visitas em 3 dias de observações (21 a 24/08/1999).

\begin{tabular}{|c|c|c|c|c|}
\hline Visitantes florais & Alimento procurado & 1ㅁ Dia & $2^{9} \mathrm{Dia}$ & $4^{\circ} \mathrm{Dia}$ \\
\hline Amazilia sp. & Néctar & ++ & 0 & + \\
\hline Phaethornis superciliosus & Néctar & - & - & 0 \\
\hline Melipona rufiventris & Pólen & ++ & ++ & ++ \\
\hline Trigona of. spinipes & Pólen & + & 0 & 0 \\
\hline Heliconius bruneyi & Néctar & - & 0 & - \\
\hline Hesperiidae & Néctar & - & 0 & - \\
\hline $\begin{array}{l}+ \text { freqüente (+ } 10 \text { visitas) } \\
+ \text { ocasional ( } 3-9 \text { visitas) } \\
- \text { raro (menos de } 2 \text { visitas) } \\
0 \text { não visitou }\end{array}$ & & & & \\
\hline
\end{tabular}

suas flores, apesar da baixa freqüência de visitas que pode ter sido influenciada pela presença dos observadores. Não se conseguiu capturar nenhuma das duas espécies de beija-flor que ao "perceberem" a rede se afastavam rapidamente.

Skutch (1952) verificou que o beija-flor Phaethornis superciliosus é o polinizador de $P$. vitifolia, embora pelo menos 20 outras espécies de beija-flores tenham ocorrido na área estudada. É o comprimento do bico como também a preferência de habitats pelos beija-flores que vão determinar o principal polinizador das flores desta Passiflora, Relações especializadas ave-flor são resultados esperados de uma longa e continuada competição interespecífica entre as aves pelo alimento floral e, entre plantas, pelas aves polinizadoras (Grant \& Grant, 1968).

Phaethornis superciliosus também foi considerado polinizador desta espécie por Fischer \& Leal (1996). Eles verificaram que a freqüência de visitas desta espécie de
Trochilidae é maior em flores com maior diâmetro do cálice que, conseqüentemente, secretam mais néctar e com isto garantem maior carga de pólen depositada nos estigmas.

As abelhas Trigona cf. spinipes e Melipona rufiventris pousam diretamente na parte dorsal das anteras e se dirigem para a parte ventral ficando de "cabeça para baixo" para a coleta de pólen e desta forma, não contactam os estigmas, estando presentes entre 07:00 e 08:00 h.

Duas espécies de borboletas pousam na plataforma formada pelo cálice e corola e introduzem a probóscide, em movimentos repetidos, entre os filetes da corona para a coleta de néctar, não contactando os estigmas e as anteras. Elas ocorreram entre 06:30 e 09:00 h.

Os resultados dos testes de sistema reprodutivo encontram-se na Tabela 2. Constatou-se que esta espécie de maracujá não é autógama e nem se reproduz por apomixia. A taxa de produção de frutos através de 
xenogamia (inter e intra-populacional) foi de $53,8 \%$. O resultado do teste de xenogamia interpopulacional, talvez seja devido a uma maior deposição de grãos de pólen e também por eles terem sido colocados nos estigmas imediatamente após à coleta das flores doadoras.

Estes resultados estão próximos aos encontrados por Ruschel, Mariot \& Orth (1999) que concluíram que a porcentagem de frutificação do maracujá-amarelo (Passiflora edulis Mart. f. flavicarpa Den.) foi de aproximadamente $54 \%$, quando visitadas pelas mamangavas e que ao protegerem as flores antes da curvatura total do estigma, estas não frutificaram.

Das 37 flores utilizadas como controle nos testes de sistema reprodutivo apenas 2 produziram frutos $(5,4 \%)$. A quantidade de frutos formados naturalmente através da contagem dos galhos de P.coccinea resultou em 18 frutos provenientes de 121 flores, ou seja, aproximadamente
$15 \%$. Esta baixa produção natural de frutos talvez esteja relacionada com a baixa freqüência de seus polinizadores (as duas espécies de beija-flores), à presença de "ladrões de néctar" (as duas espécies de Lepidoptera) que diminuem a oferta de alimento para os polinizadores e à presença das duas espécies de Hymenoptera, que diminuem a quantidade de pólen para que ocorra a formação de frutos.

As medidas dos frutos resultantes dos testes de sistema reprodutivo e dos coletados na área de estudo encontramse na Tabela 3. Os menores resultados foram obtidos nos frutos provenientes das flores utilizadas como controle e nos quatro frutos coletados na área de estudo (aberta), confirmando a necessidade de mais visitas dos polinizadores para fecundar um maior número de óvulos.

\section{Agradecimentos}

Agradeço aos professores do

Tabela 2. Resultados dos testes de sistema reprodutivo de Passiflora coccinea. ( $\mathrm{N}^{\circ}=$ número)

\begin{tabular}{lccc}
\hline TRATAMENTO & $\begin{array}{c}\text { No DE FLORES } \\
\text { TESTADAS }\end{array}$ & $\begin{array}{c}\text { No DE FRUTOS } \\
\text { OBTIDOS }\end{array}$ & $\begin{array}{c}\text { SUCESSO } \\
(\%)\end{array}$ \\
\hline $\begin{array}{l}\text { CONTROLE } \\
\begin{array}{l}\text { XENOGAMIA INTRA- } \\
\text { POPULACIONAL }\end{array}\end{array}$ & 37 & 02 & 5,4 \\
$\begin{array}{l}\text { XENOGAMIA INTER- } \\
\text { POPULACIONAL }\end{array}$ & 02 & 05 & 45,5 \\
$\begin{array}{l}\text { APOMIXIA } \\
\text { AUTOGAMIA }\end{array}$ & 05 & 02 & 100,0 \\
$\begin{array}{l}\text { AUTOGAMIA } \\
\text { INDUZIDA }\end{array}$ & 15 & 00 & 0 \\
TOTAL & 04 & 00 & 0 \\
\hline
\end{tabular}


Tabela 3. Medidas dos frutos provenientes dos testes de sistema reprodutivo e coletados na área de estudo (polinização aberta) de Passiflora coccinea. ( $\left(\mathrm{N}^{\circ}=\right.$ número)

\begin{tabular}{lcccc}
\hline TRATAMENTO & $\begin{array}{c}\text { PESO } \\
(\mathrm{g})\end{array}$ & $\begin{array}{c}\text { COMPRIMENTO } \\
(\mathrm{cm})\end{array}$ & $\begin{array}{c}\text { LARGURA } \\
(\mathrm{cm})\end{array}$ & $\begin{array}{c}\text { No SEMENTES } \\
\text { (média) }\end{array}$ \\
\hline CONTROLE & $8,3 \pm 4,9$ & $4,0 \pm 0,6$ & $3,1 \pm 0,3$ & $101,0 \pm 15,5$ \\
$\begin{array}{l}\text { XENOGAMIA INTRA- } \\
\text { POPULACIONAL }\end{array}$ & $19,6 \pm 8,2$ & $4,1 \pm 0,4$ & $3,4 \pm 0,5$ & $273,4 \pm 78,2$ \\
$\begin{array}{l}\text { XENOGAMIA INTER- } \\
\text { POPULACIONAL }\end{array}$ & $22,6 \pm 2,5$ & $4,7 \pm 0,1$ & $3,5 \pm 0,1$ & $384,5 \pm 112,4$ \\
$\begin{array}{l}\text { ABERTA } \\
\text { TOTAL }\end{array}$ & $12,9 \pm 6,1$ & $4,2 \pm 1,0$ & $2,9 \pm 0,7$ & $162,5 \pm 103,8$ \\
& $16,3 \pm 7,7$ & $4,2 \pm 0,6$ & $3,2 \pm 0,5$ & $229,8 \pm 121,9$ \\
\hline
\end{tabular}

curso de Biologia Reprodutiva do INPA - 1999, Dr. Antônio Carlos Webber e Dr. Rogério Gribel pelos ensinamentos; à Aldenora, ao Manoel e ao Paulo pela colaboração durante o trabalho de campo, à pesquisadora Dra. Catarina Motta (CPEN - INPA), pela determinação de uma das espécies de Lepidoptera, ao Dr. Charles Clement (CPCA - INPA) pela correção do abstract e ao Atilio Storti Filho, pelo apoio constante.

\section{Bibliografia citada}

Amela-Garcia, M.T.; Hoc, P.S. 1998. Flower biology of Passiflora foetida (Passifloraceae). Rev. Biol. Trop., 46(2): 191-201.

Arnold, R.M. 1982. Floral biology of Chaenorrhinum minus (Scrophulariaceae) a self-compatible annual. Amer. Midl. Nat., 108(2): 317-324. (apud Kearns, C.A.; Inouye, D.W. 1993. Techniques for pollination biologists. University Press of Colorado, Colorado).

Carreira, L.M.M. 1978. Aspectos da ultraestrutura do pólen de Passiflora coccinea Aubl. (Passifloraceae). Acta Amazonica, 7(3): 329-332.

Cruden, R.W.; Hermann, S.M. 1983. Studying nectar? Some observations on the art. In
Bentley, B.; Elias, T. (eds.). The biology of nectaries. Columbia University Press, New York. cap 8. p: 223-241.

Dafni, A, 1992. Pollination ecology. A practical approach. IRL Press, Oxford. 250p.

Da Silva, M.M.; Bruckner, C.H.; Picanço, M.; Molina-Rugama, A.J. 1999. Número floral, clima, densidad poblacional de Xylocopa spp. (Hymenoptera: Anthophoridae) y polinización del maracuyá (Passiflora edulis f. flavicarpa). Rev. Biol. Trop., 47(4): 711-718.

Fischer, E.A.; Leal, I.R. 1996. Efeito da taxa de produção de néctar na polinização de flores de Passiflora coccinea, Amazônia Central. Resumos do XLVII Congresso Nacional de Botânica, Nova Friburgo, RJ. p. 408 .

Gertz, O. 1938. Ueber die Verbreitung des Anthoclors bei den Compositen. $\mathrm{Kgl}$. Fysiogr: Sällsk. Lund. Förh., 8: 62-70 (apud Scogin, R.; Young, D.A.; Jones, Jr., C.E. 1977, Anthochlors pigments and pollination biology. II. The ultraviolet floral pattern of Coreopsis gigantea (Asteraceae). Bull. Torrey Bot. Club, 104(2): 155-159).

Grant, K.A.; Grant, V. 1968. Hummingbirds and their flowers. Columbia University Press, New York. 103p.

Heywood,V.H. 1985. Las plantas com flores. Editorial Reverté, Barcelona. 332p. 
Percival, M. 1974. Floral ecology of a coastal scrub in southeast Jamaica. Biotropica, 6(2): 104-129.

Pio Corrêa, M. 1978. Dicionário das planias úteis do Brasil e das exóticas cultivadas. Ministério da Agricultura, Rio de Janeiro. Volume 5: 125.

Radford, A.E.; Dickison, W.C.; Massey, J.R.; Ritchie Bell, C. 1974. Vascular plant systematics. Harper \& Row Publish, New York. $891 \mathrm{p}$.

Ruschel, A.R.; Mariot, A.; Orth, A.I. 1999. Observações sobre a polinização natural do maracujá amarelo (Passiflora edulis Mart. f. flavicarpa Den.) em Florianópolis, SC. L Congresso Nacional de Botânica. Blumenau, SC. p.207.

Sazima, I.; Sazima, M. 1989. Mamangavas e irapuás (Hymenoptera, Apoidea): visitas, interações e' conseqüências para a polinização do maracujá (Passifloraceae). Revta. Bras. Ent., 33(1): 109-118.

Sazima, M.; Sazima, I. 1978. Bat pollination of the passion flower, Passiflora mucronata in southeastern Brazil. Biotropica, 10(2): 100-109.

Sazima, M.; Sazima, I. 1987. Additional observations on Passiflora mucronata, the batpollinated passionflower. Ci. e Cult., 39(3): $310-312$

Skutch, A.F. 1952. Scarlet passion flower. $\mathrm{Na}$ ture Mag., 45: 523-525, 550. (apud Grant, K.A.; Grant, V. op. cit.).

Vanderplank, J. 1996. Passion flower. The MIT Press, Cambridge. p.70-71.0

Aceito para publicação em 15/07/2002 